\section{Anomalous Bifurcation of Wirsung's Duct in a Child as a Cause of Recurrent Pancreatitis}

Endoscopic retrograde cholangiopancreatography (ERCP) allows clearer identification of congenital anomalies of the pancreas $(1,2)$ which have been implicated as causes of pancreatitis in children and adults (3).

A 6-year-old boy was admitted to the emergency room due to abdominal pain, predominantly on the left side. For the previous two years, he had been suffering from episodes of recurrent abdominal pain. In all instances, abdominal tenderness, leukocytosis, and normal serum amylase levels were found. No history of drug intake or abdominal trauma was reported. The ultrasonographic examination did not show any abnormalities. The amylase/creatinine clearance ratio was $4.25 \%$ (normal value $3 \%$ ). A pancreatic test (i.v. morphine $0.15 \mathrm{mg} / \mathrm{kg}$ body weight and neostigmine $0.04 \mathrm{~g} / \mathrm{kg}$ body weight), with hourly measurement of the serum amylase level, showed a six-fold increase in baseline values. ERCP findings are shown in Figure 1. At surgery, the ERCP findings were confirmed, and the anomalous segment running from the tail of the pancreas to the posterior aspect of the gastric antrum was removed. The diagnosis of chronic pancreatitis with a pseudocyst of $2.0-2.5 \mathrm{~cm}$ was confirmed by the pathologic study.

Pancreas divisum and other abnormalities are generally associated with episodes of pancreatitis (4). In the case presented here, an anomalous bifurcation of Wirsung's duct and morphological features of chronic pancreatitis were detected by ERCP. The changes were localized in the anomalous segment, which after the bifurcation ran to the posterior gastric wall, where a pseudocystic cavity was seen. Kikuchi et al. (5) reported a similar pancreatic duct abnormality, in which the bifurcation of Wirsung's duct manifested as a submucosal gastric tumor. In this case, morphological appearance of both ducts was normal, and no specific therapy was required. ERCP was of considerable value in establishing the diagnosis, and the procedure was successfully carried out using the conventional adult fiber endoscope.

\author{
E. Meroño ${ }^{1}$, A. Martinez-Pardo ${ }^{2}$, V.Morales ${ }^{3}$, V.F.Moreira ${ }^{1}$ \\ J. C. Erdozain', C. Martin-de-Argila' \\ ${ }^{1}$ Dept. of Gastroenterology \\ ${ }^{2}$ Dept. of Pediatrics \\ ${ }^{3}$ Dept. of General Surgery, Ramón y Cajal Hospital, Madrid, \\ Spain
}

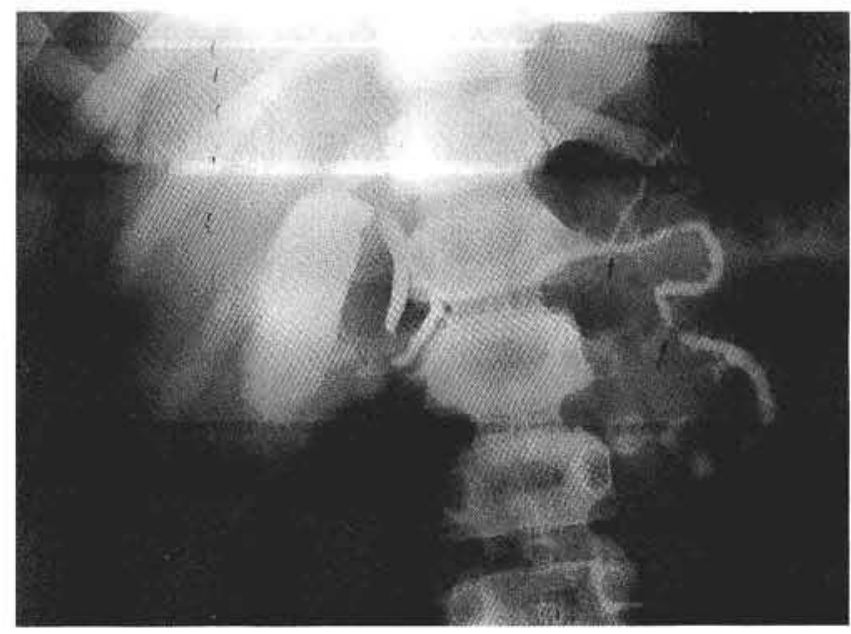

Figure 1: Anomalous bifurcation of Wirsung's duct at the distal third of the body of the pancreas. The bifurcated branch runs medially. The anomalous duct shows a stricture at the bifurcation level and in its middle portion (arrows), is dilated, shows microcysts in its ductules, and ends in a pseudocystic cavity. The remaining pancreatic gland and the biliary tree have a normal appearance

\section{References}

1. Cotton PB. Congenital anomaly of pancreas divisum as a cause of obstructive pain and pancreatitis. Gut 1980;21: 105-14.

2. Chevillote G, Sahel J, Raillat A, Sarles H. Annular pancreas: report of one case associated with acute pancreatitis and diagnosed by endoscopic retrograde pancreatography. Dig Dis Sci 1984; 29: $75-7$.

3. Yeldin ST, Dubois RS, Philippart AI. Pancreas divisum: a cause of pancreatitis in childhood. J Pediatr Surg 1984; 19: 793-4.

4. Meroño Garcia E, Moreira V, Ruiz del Arbol L, Suarez E, Faro MV, Garcia Plaza A. Pancreas divisum: frecuencia en nuestro medio. Rev Esp Enferm Dig 1981; 59: 313-8.

5. Kikuchi K, Nomiyama T, Miwa M, Harasawa S, Miwa T. Bifid tail of the pancreas: a case presenting as a gastric submucosal tumor. Am J Gastroenterol 1983; 78: 23-7.

Corresponding Author

J. C. Erdozain, M.D.

Condesa del Venadito 20,6B

28027 Madrid

Spain

Fax: $+34-1-3739206$ 